\title{
BMJ Open Exercise in older women with breast cancer during systemic therapy: study protocol of a randomised controlled trial (BREACE)
}

\begin{abstract}
Høgni Hammershaimb Andersen (D) , ${ }^{1,2}$ Marta Kramer Mikkelsen, ${ }^{2,3}$ Ida Lundager, ${ }^{1}$ Cecilia Margareta Lund, ${ }^{4}$ Julia Sidenius Johansen, ${ }^{2,4,5}$ Anders Vinther, ${ }^{1,6}$ Carsten Bogh Juhl, ${ }^{7,8}$ Bo Zerahn, ${ }^{9}$ Anne-Mette Ragle, ${ }^{1}$ Dorte Lisbet Nielsen ${ }^{2,5}$
\end{abstract}

To cite: Andersen $\mathrm{HH}$, Mikkelsen MK, Lundager I, et al. Exercise in older women with breast cancer during systemic therapy: study protocol of a randomised controlled trial (BREACE). BMJ Open 2020;10:e038674. doi:10.1136/ bmjopen-2020-038674

- Prepublication history and additional material for this paper are available online. To view these files, please visit the journal online (http://dx.doi. org/10.1136/bmjopen-2020038674).

Received 19 March 2020 Revised 05 August 2020 Accepted 12 August 2020

Check for updates

(c) Author(s) (or their employer(s)) 2020. Re-use permitted under CC BY-NC. No commercial re-use. See rights and permissions. Published by BMJ.

For numbered affiliations see end of article.

\section{Correspondence to} Høgni Hammershaimb Andersen;

hoegni.hammershaimb. andersen@regionh.dk

\section{ABSTRACT}

Introduction Exercise interventions have been widely investigated in patients with cancer and demonstrate beneficial effects. However, intervention studies that include older women with breast cancer exercising during medical treatment are scarce. Hence, the aim of this study is to investigate the effect of a 12-week exercise-based intervention in older women ( $\geq 65$ years) with breast cancer receiving (neo)adjuvant or first-line or second-line systemic therapy.

Methods and analysis This is a single-centre, twoarmed randomised controlled trial. We anticipate including 100 patients, who will be randomised 1:1 to exercisebased intervention or control stratified by treatment setting ((neo)adjuvant or metastatic) and treatment (chemotherapy or endocrine therapy + cyclin-dependent kinase (CDK) $4 / 6$ inhibitors). The intervention group will receive standard oncological treatment and a 12-week supervised exercisebased intervention comprising a progressive resistance exercise programme two times per week, a protein supplement after exercise and a home-based walking programme based on daily step counts. The control group will receive standard oncological treatment. Assessments will be performed at baseline and 6,12 and 24 weeks after start of the intervention. Primary outcome is physical function, measured by the 30-second Chair Stand Test. Secondary outcomes are feasibility (compliance and adherence to intervention), objective and patient-reported functional measures (6-meter and 10-meter gait speed; 6-min Walk Test; Handgrip Strength; Stair Climb Test; Physical Activity Questionnaire), symptom burden and well-being (MD Anderson Symptom Inventory; Hospital Anxiety and Depression Scale), quality of life (European Organization for Research and Treatment of Cancer Quality of Life Questionnaire Core-30 and B23), body composition (dual-energy X-ray absorptiometry scan), side effects, inflammatory biomarkers, hospitalisation and survival. Ethics and dissemination The protocol was reviewed and accepted by the Scientific Ethics Review Committee of the Capital Region of Denmark, 17 June 2018 (VEK ref.: $\mathrm{H}-18021013)$. Trial results will be submitted for publication in a peer-reviewed journal and presented on conferences, in oncology wards, exercise centres in municipalities and patient organisations, ensuring dissemination to relevant stakeholders.
Strengths and limitations of this study

- This study includes an evidence-based exercise intervention, according to recommendations from the American College of Sports Medicine.

- The study includes a broad array of outcome measures for patients with breast cancer, including physical function, fatigue, quality of life and patient reported physical and mental well-being.

- The physiotherapists conducting the testing of participants in this study are blinded.

- It is not possible to blind the participants and the physiotherapists, delivering the exercise intervention.

Trial registration number https://clinicaltrials.gov/ on 3 September 2018. Identifier: NCT03656731.

\section{INTRODUCTION}

\section{Cancer and age}

Breast cancer is the most frequent type of cancer among women and is a major cause of cancer-related disability and deaths worldwide. It is estimated that about 2.1 million new cases of breast cancer in women occurred worldwide in $2018 .{ }^{1}$

The risk of breast cancer increases with age. The median age at time of diagnosis is 61 years, and $30 \%$ of patients with breast cancer are $>70$ years. ${ }^{2}$ Ageing is closely associated with comorbidity. ${ }^{34}$ More than $50 \%$ of older cancer survivors have a comorbidity, and more than four chronic conditions are seen in $25 \%$ of older women with breast cancer $>65$ years. ${ }^{3}$ These conditions often lead to functional impairment; poorer survival and a decline in quality of life (QoL) and mental, physical and social health. ${ }^{3} 4$ Compared with an age-matched population without a history of cancer, the prevalence of the above-mentioned impairments is significantly 
higher in cancer survivors: limitations in activities of daily living (ADL) $26.9 \%$ versus $31.9 \%$, poor or fair self-rated health $20.9 \%$ versus $27.4 \%$ and prevalence of geriatric syndromes $53.9 \%$ versus $60.8 \%$, respectively. ${ }^{56}$ Furthermore, the negative effects of primary sarcopaenia, defined as age-related loss of muscle mass, ${ }^{7}$ may increase in patients with cancer due to cancer-related cachexia, resulting in decreased treatment tolerability, increased risk of postoperative complications and poorer survival. ${ }^{89}$ In women with breast cancer, antineoplastic treatment may increase muscle atrophy and decrease mitochondrial function, which also have a negative effect on survival. ${ }^{10}$ Although breast cancer among older women is associated with a more favourable tumour biology, Danish patients with breast cancer over the age of 70 years have a poorer prognosis and increased mortality compared with younger patients. ${ }^{11}$

\section{Exercise interventions}

Exercise interventions have been shown to be effective in preventing or relieving cancer symptoms and side effects from cancer treatment. ${ }^{12}$ Furthermore, several studies including different exercise interventions (eg, aerobic exercise or resistance exercise) have shown improved physical and mental health, QoL, muscle strength, physical function and reduced fatigue in patients with cancer. $^{13-15}$

Nevertheless, very few trials have investigated the effect of exercise interventions in older women with breast cancer receiving chemotherapy or therapy for advanced/ metastatic disease. ${ }^{1617}$

This article describes the protocol of the Breast Cancer Exercise (BREACE) study. The Standard Protocol Items: Recommendations for Interventional Trials checklist for interventional trials and the Consensus on Exercise Reporting Template will be used as reporting guidelines. ${ }^{1819}$

\section{METHODS AND ANALYSIS \\ Aim}

The aim of this study is to investigate the feasibility and effect of an exercise-based intervention among older women with breast cancer receiving antineoplastic treatment.

\section{Hypothesis}

We hypothesise that (1) the exercise intervention in this trial will improve muscle strength and physical function, decrease weight loss and muscle wasting, reduce symptoms and side effects from the medical treatment, and improve QoL, mental, and physical health compared with the control treatment; (2) the recruitment of the patients will be difficult, especially because of side effects to medical treatment and age-related burdens and (3) a considerable attrition rate will occur, which is in line with prior studies investigating the effect of exercisebased interventions in older cancer survivors, which have
Figure 1

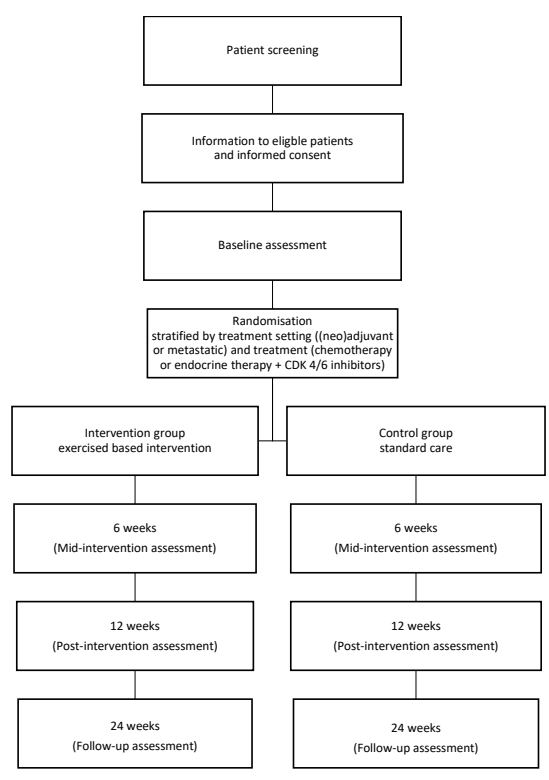

Figure 1 Study design for the Breast Cancer Exercise study, from patient screening to 24 weeks of follow-up.

experienced relatively high numbers of dropouts during intervention.

However, we expect that the intervention will be feasible and effective due to it targeting the specific needs of the older patient with breast cancer treated with (neo)adjuvant or first-line or second-line palliative therapy and that it can be integrated into the future treatment of this group in both hospital and home settings.

\section{Design}

BREACE is one of the five studies in the TOLCA project(Translational and clinical research focused on older patients with cancer), which aims to improve patient survival and QoL. BREACE is a single-centre, twoarmed randomised controlled trial. Intervention time will be 12 weeks, mainly comprising a progressive resistance exercise intervention for older women ( $\geq 65$ years) with breast cancer treated with neo or adjuvant or first-line or second-line palliative systemic therapy at the Department of Oncology at Copenhagen University Hospital, Herlev and Gentofte, Denmark (figure 1). A minimum of 29 evaluable patients will be included in each group, based on a sample size calculation. Since some patients are expected to drop out before evaluation, we anticipate inclusion of up to 100 participants in the study.

The BREACE study is an ongoing study. The first participant was included on 15 November 2018. Recruitment is currently planned until 31 August 2021. 


\section{Participants}

Due to low inclusion rates, an amendment to the protocol was made, broadening the inclusion criteria. Age at entry was lowered from $\geq 70$ years to $\geq 65$ years. In addition to adjuvant treatment, neoadjuvant and first-line to secondline treatments were also included.

\section{Patients eligible}

Patients eligible for inclusion in this trial must (1) be diagnosed with primary breast cancer and scheduled for neoadjuvant chemotherapy, \pm human epidermal growth factor receptor 2 (HER2) inhibitor, tantihormonal treatment; (2) have undergone surgery for primary breast cancer within 12 weeks and received adjuvant chemotherapy, \pm HER2 inhibitor, \pm antihormonal treatment; (3) have advanced or metastatic breast cancer and receive first-line or second-line palliative chemotherapy, \pm HER2 inhibitor, or antihormonal treatment, +cyclin-dependent kinase (CDK) 4/6 inhibitor; (4) have a performance status (PS) score $\leq 2$ (based on the Eastern Cooperative Oncology Group (ECOG)), see online supplemental file 1 ; (5) be $\geq 65$ years of age at the time of signing the informed consent form, see online supplemental file 2; and (6) speak and read Danish.

\section{Exclusion criteria}

Exclusion criteria: (1) conditions preventing participation in the exercise intervention, including, but not restricted to, severe pain and physical and/or mental impairments; (2) documented and uncontrolled brain metastases; (3) coexisting types of cancer; (4) cognitive impairments that hinder informed consent; (5) medical conditions that could be negatively affected by the intervention including, but not restricted to, congestive heart failure (NYHA class III-IV), unstable angina pectoris, implantable cardioverter defibrillator or myocardial infarction within 6 months and (6) bone metastasis which could increase the risk of injuries caused by the exercise intervention.

The primary investigator will identify potentially eligible patients, and an oncologist will assess the eligibility of patients based on the inclusion and exclusion criteria. Eligible patients will hereafter be informed about the study and asked whether they would be interested in further contact. If interested, patients will be contacted, thoroughly informed about the study and their rights in light of the Declaration of Helsinki, and potentially included by the primary investigator.

\section{Randomisation}

The allocation sequence will be developed by a statistician with no involvement in the study. Patients included in the study will be randomised 1:1 to either the intervention group or the control group. Stratification will be performed at randomisation by cancer stage ((neo) adjuvant or metastatic), and treatment (chemotherapy or endocrine therapy $\pm \mathrm{CDK} 4 / 6$ inhibitors) as this is expected to have an influence on the results. Randomisation and

\begin{tabular}{ll}
\hline $\begin{array}{l}\text { Duration of exercise } \\
\text { program }\end{array}$ & $\begin{array}{l}60 \mathrm{~min} . \text { (warm-up } 15 \mathrm{~min} ., \mathrm{PRT} 35 \mathrm{~min} ., \text { cool } \\
\text { down/stretching } 10 \mathrm{~min} .)\end{array}$ \\
\hline No. of repetitions (PRT) & $\begin{array}{l}15-12 \mathrm{RM} \text { (week 1-3), } 12 \text { RM (week 4-6), } 10 \\
\text { RM (week 7-9), 8 RM (week 10-12) }\end{array}$ \\
\hline No. of sets (PRT) & 2 (week 1-3), 3 (week 4-12) \\
\hline Resting period between & $40-60$ sec. \\
sets (PRT) & \\
\hline No. of exercise sessions & 2 per week \\
\hline Resting period between & $2-4$ days \\
sessions &
\end{tabular}

Intervention period 12 weeks

\begin{tabular}{ll}
\hline Description of warm-up & Exercises performed with low to moderate \\
& intensity, including a combination of balance \\
& and flexibility (e.g. walking/jogging, shoulder \\
& specific flexibility exercises, balance boards, \\
& lunges etc.)
\end{tabular}

Description of PRT

exercises

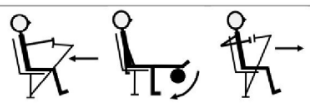

low row, knee flexion, chest press,

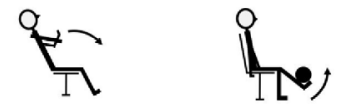

abdominal crunch, knee extension,

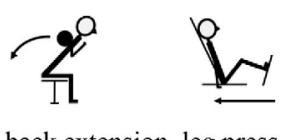

back extension, leg press.

Figure 2 A detailed description of the supervised exercise programme. PRT, progressive resistance training; RM, repetition maximum.

stratification will be performed through the randomisation module in the web-based research platform Research Electronic Data Capture.

\section{Intervention}

Acknowledging exercise recommendations from the Cancer and Aging Group, ${ }^{16}$ patients in the intervention group will receive an exercise-based intervention in addition to standard oncological care. The intervention will comprise a 12-week supervised group-based exercise programme, mainly focusing on progressive resistance training (PRT) (figure 2), and a nutrition supplement (eg, protein drink). In addition, an individual lightto-moderate home walking programme, based on step counts and monitored by a pedometer, is included to avoid inactivity. The PRT and walking programme will be individually tailored by experienced physiotherapists who will consider any relevant comorbidities and/or cancerrelated (eg, bone metastasis), motivational, physical or psychological factors.

The 12-week supervised group-based intervention will comprise participation in the supervised exercise programme two times per week (60 min/session). The 
exercise programme will include a warm-up session, focusing mainly on light-to-moderate intensity exercise, flexibility and balance exercises recommended and tailored for this group ${ }^{20}(15 \mathrm{~min})$, a PRT programme performed on seven resistance training machines targeting the large muscle groups (low row, knee flexion, chest press, abdominal crunch, knee extension, back extension and leg press (35 min)) (figure 2), followed by cool down, stretching and relaxation $(10 \mathrm{~min})$. The PRT will be initiated with low load and high repetition numbers and progresses in week 3 from 2 to 3 sets and from 15 to 8 repetition maximum during the 12-week intervention (figure 2). The PRT follows recommendations from the American College of Sports Medicine (ACSM) ${ }^{20-22}$ If necessary, a conservative approach to the recommendations for PRT for older adults will be used, based on assessment by experienced physiotherapists. ${ }^{20}{ }^{22}$

The supervised exercise training will take place at the Department of Physiotherapy and Occupational Therapy at Herlev and Gentofte Hospital and be led by experienced physiotherapists. Furthermore, patients in the intervention group will receive a 'replacement training programme' comprising a few effective exercises for the major muscle groups that can be performed at home. If a patient cancels a group-based training session during the intervention period, the patient will be instructed to perform the exercises in the replacement programme instead. Patients will be thoroughly introduced to these replacement exercises when entering the study. A nutritional supplement (protein drink) is offered after each supervised exercise session, containing 8 or $12 \mathrm{~g}$ of protein and $300 \mathrm{kcal}$, consumed immediately after the exercise session, supervised by the physiotherapists. Furthermore, a walking programme tailored individually to each patient and based on daily step counts, measured by a GARMIN vivofit V.4 wristwatch, will be included. The mean of 3 days step count measured pre intervention will be used as starting point. A weekly goal for each patient will be set once a week by the patient and the primary investigator. Although the goal is to increase the patient's physical activity, the weekly goal can be increased, maintained or decreased from the previous week, based on motivation, physical and psychological state, which could be negatively affected by the cancer treatment.

\section{Control group}

Patients randomised to the control group will receive standard oncological care, including information about physical activity which will not be restricted, due to ethical considerations.

\section{Assessments}

Data collection and test for both groups will be performed at baseline, midintervention (6 weeks), post intervention (12 weeks) and follow-up (24 weeks) (table 1).

To minimise dropouts, the exercise intervention will be supervised, and participants exercise in groups with a possibility to interact and socialise with each other. In addition, the primary investigator will be present, but not involved, in all test seances for all participants, whenever possible. Furthermore, exercise and test will be coordinated with other visits to the hospital to avoid additional hospital visits.

\section{Primary outcome}

The primary outcome is the 30-second Chair Stand Test (30s-CST) and will be used to measure physical function and lower extremity strength (see online supplemental file 3). The 30s-CST is deemed valid and reliable to measure lower body strength and function among healthy older adults ${ }^{23}$ and has been used and tested in patients with cancer. ${ }^{24}$ Furthermore, the 30s-CST has frequently been used in measuring physical capacity in older people and as an indicator for functional independence. ${ }^{23}$

\section{Secondary outcomes}

\section{Feasibility measures}

Feasibility measures (acceptability, attrition and adherence to the study) will be evaluated by attendance rate to the intervention in \%. Reasons for declining participation or dropout will be obtained by asking the patients.

\section{Physical function}

A 6-meter gait speed test and 10-meter gait speed test at normal and maximum gait speed will be used to measure physical performance, the 6-minute Walk Test to measure physical capacity and endurance, the Handgrip Strength Test (Grip SAEHAN DHD-1 Digital Hand Dynamometer) to measure physical function of the upper body, and a Stair Climb Test to measure lower extremity muscle power (further descriptions of the physical function tests in online supplemental file 3).

\section{Patient-reported outcome measures}

Patient-reported outcome measures will mainly comprise questionnaires measuring symptom burden (MD Anderson Symptom Inventory (MDASI)), QoL (The European Organization for Research and Treatment of Cancer Quality of Life Questionnaire C30 and the breast cancer-specific module), depression and anxiety (The Hospital Anxiety and Depression Scale) and physical activity level (Saltin-Grimby Physical Activity Level Scale). Patient-reported PS will be assessed using the ECOG Scale.

\section{Therapy tolerance}

Therapy tolerance will be registered as completion of scheduled (chemo) therapy, dose reductions, treatment discontinuations and toxicity based on information obtained from patient records.

\section{Body composition}

Whole-body dual-energy X-ray absorptiometry scan and bioelectrical impedance will be used to assess body composition and hydration status and include data on weight, height and body mass index (BMI). 
Table 1 Overview of schedule of assessments

\begin{tabular}{|c|c|c|c|c|}
\hline & $\begin{array}{l}\text { Inclusion } \\
\text { (baseline) }\end{array}$ & $\begin{array}{l}\text { Midway } \\
\text { (6 weeks) }\end{array}$ & $\begin{array}{l}\text { Post intervention } \\
\text { (12 weeks) }\end{array}$ & $\begin{array}{l}\text { Follow-up } \\
\text { (24 weeks) }\end{array}$ \\
\hline Informed consent (Breast Cancer Exercise) & $\mathrm{X}$ & & & \\
\hline Clinical and medical data & $x$ & $x$ & $x$ & $x$ \\
\hline Demographics & $\mathrm{x}$ & & & \\
\hline \multicolumn{5}{|l|}{ Feasibility measures } \\
\hline Recruitment rates & $x$ & & & \\
\hline Retention & $x$ & $x$ & $\mathrm{X}$ & $x$ \\
\hline Adherence & & $x$ & $x$ & \\
\hline MDASI & $x$ & $x$ & $x$ & $x$ \\
\hline EORTC QLQ-C30 and BR23 & $\mathrm{x}$ & $x$ & $\mathrm{X}$ & $x$ \\
\hline HADS & $x$ & $x$ & $x$ & $x$ \\
\hline \multicolumn{5}{|l|}{ Physical tests } \\
\hline 30s-CST (primary outcome) & $x$ & $x$ & $x$ & $x$ \\
\hline 6MWT & $x$ & $x$ & $\mathrm{X}$ & $x$ \\
\hline $6 m G S$ & $x$ & $x$ & $x$ & $x$ \\
\hline $10 \mathrm{mGS}$ & $x$ & $x$ & $x$ & $x$ \\
\hline HST & $x$ & $x$ & $x$ & $x$ \\
\hline Weight, BMI & $x$ & $x$ & $x$ & $x$ \\
\hline DXA scan & $X$ & & $x$ & \\
\hline $\mathrm{BI}$ & $X$ & & $x$ & \\
\hline
\end{tabular}

*For patients with breast cancer who accept participation in the PRIMA B trial (Prospective collection of blood samples from patients with breast cancer).

$\mathrm{BI}$, bioimpedance measurement; BMI, body mass index; CARG, Cancer and Aging Research Group; CCI, Charlson Comorbidity Index; DXA, dual-energy X-ray absorptiometry; ECOG, Eastern Cooperative Oncology Group; EORTC QLQBR23, European Organization for Research and Treatment of Cancer Breast Cancer Specific Quality of Life Questionnaire; EORTC QLQ-C30, European Organization for Research and Treatment of Cancer Quality of Life Questionnaire Core-30; HADS, Hospital Anxiety and Depression Scale; HST, Handgrip Strength Test; MDASI, MD Anderson Symptom Inventory; 6mGS, 6-meter gait speed; 10mGS, 10-meter gait speed; 6MWT, 6-minute Walk Test; PROMs, patient reported outcome measures; PRPS, patient-reported performance status; 30s-CST, 30-second Chair Stand Test; SCT, Stair Climb Test.

\section{Biomarkers}

Overall, $30 \mathrm{~mL}$ blood will be drawn at baseline and 6 , 12 and 24 weeks after start of the intervention. Serum C reactive protein (CRP) (liquid assay reagent by an immunoturbidimetric method on a fully automated chemistry analyser from Kit-test SENTINEL CRP Ultra), interleukin 6 (ELISA from Quantikine HS600B, R\&D Systems, Abingdon, UK), YKL-40 (ELISA from Quidel, San
Diego, California, USA), growth differentiation factor 11 (GDF11), and GDF15 will be analysed. Furthermore, 92 proteins associated with inflammation, immunology and cancer will be analysed using the Olink immuno-oncology protein panel (www.olink.com), a multiplex immunoassay using proximity extension assay. Blood samples and data on biomarkers will be obtained only in patients who have also accepted participation in the PRIMA B (Prospective 
collection of bloodsamples from patients with breast cancer) biomarker trial (VEK ref.: KA-H-3-2014-120).

\section{Hospital admissions}

Hospital admissions will include length of stay and numbers and causes of hospital admissions. All contacts to the emergency room will be registered.

\section{Mortality/survival}

Incidences of deaths and cause of death (cancer related and non-cancer related) will be registered throughout the study period.

\section{Data registration}

\section{Clinical and medical data}

Medical records will be used to obtain patient data on time of breast cancer diagnosis, disease stage, side effects, location of metastases, type of treatment, comorbidities (assessed by Charlson Comorbidity Index and PS (ECOG). Calculation of the risk of systemic therapy toxicity will be based on the Cancer and Aging Research Group toxicity score with data from medical records and patient questionnaire.

\section{Demographic and patient-reported data}

Patient questionnaire and medical records will be used to obtain data including working and civil status, educational level, smoking and alcohol habits, weight loss prior to diagnosis or progression and during the study (kg/percentage), and prior and current physical activity level.

\section{Blinding procedure}

All physical tests, including the 30s-CST (primary outcome), will be conducted by the same two physiotherapists blinded for group allocation, and thus not involved in delivering the exercise intervention. A standardised test manual will be used to ensure uniformity.

\section{Sample size}

The sample size calculation is based on a minimal clinical important improvement (MCII) for the 30s-CST of 2.6 repetitions and an SD of 3. The SD is based on the previous studies using the 30s-CST in patients with advanced cancer. ${ }^{25}{ }^{26}$ An MCII for the 30s-CST has not yet been established in patients with cancer but is suggested to be 2.6 in patients with osteoarthritis. ${ }^{27}$ With a type I error of $5 \%$ and a power of $90 \%$, a total of 29 patients in each group are needed to be able to detect a betweengroup difference of 2.6 repetitions in the 30s-CST after the intervention (12 weeks).

The dropout rate is unknown in older patients with breast cancer. If the dropout rate is $40 \%$ throughout the study period, we anticipate it will be necessary to include up to a total of 100 patients.

\section{Statistical analyses}

Baseline characteristics will be presented separately for the intervention and control groups. Data from the results of the physical tests, body composition measures, blood samples and questionnaires will be reported as mean and 95\% CI or as median and IQR, as appropriate. Feasibility measures (attrition, adherence, acceptability and adverse events) will be presented as numbers and percentages. Reasons for declining participation and dropouts will be described, if stated. Differences between groups in continuous data will be analysed using a repeated measurement analysis, adjusted for baseline scores and covariates (ie, treatment, physical activity, comorbidities and BMI) and allowing for interaction between time and intervention. Analysis of time to death (by cancer or other causes) will be carried out using the Kaplan-Meier estimator, competing risk analyses and Cox regression analyses. Between-group comparisons will be carried out using Grey's test and the log-rank test. The overall risk of death will be estimated by a Cox proportional hazard regression and the cancer-related cause of death by causespecific Cox proportional hazard regression. The significance level of all tests is set a $p<0.05$, and analyses will be carried out in SAS 9.4, STATA or R by a statistician in collaboration with the primary investigator.

\section{Data statement}

Technical appendix, statistical codes and dataset will be available at any time by contacting the corresponding author.

\section{Patient and public involvement}

Patients and/or public were not involved in designing this study.

\section{ETHICS AND DISSEMINATION}

The study protocol has been peer reviewed and approved by the Scientific Ethics Review Committee of the Capital Region of Denmark (VEK ref.: H-18021013). Any protocol modifications will be communicated to this Committee.

Due to ethical consideration on information burden, participants will be informed at their second visit to the oncological ward. Informed oral and written consent will be obtained from all participants before inclusion.

The intervention is not expected to cause adverse events and any potential adverse events are believed to be outweighed by the beneficial effects of the intervention.

Trial results will be communicated to all participants, if requested, disseminated by publications in peer-review journals and presented at relevant conferences.

\section{DISCUSSION}

Exercise-based intervention studies that include older patients with breast cancer receiving medical treatment are scarce, ${ }^{16}$ although physical activity is regarded as a safe and effective intervention in improving health-related QoL, as well as physiological, behavioural and physical outcomes among breast cancer survivors. ${ }^{28}$ Only a small number of minor adverse events have been reported, 
and there is no evidence that physical activity interventions among women diagnosed with breast cancer can have harmful or negative effects. ${ }^{28}$ Exercise interventions among patients with breast cancer receiving medical treatment that include resistance training or a combination of resistance and aerobic training ${ }^{29-39}$ have been shown to reduce fatigue, ${ }^{31} 3439$ symptoms of depression ${ }^{31}$ and improve QoL. ${ }^{30} 3{ }^{34}$ Furthermore, exercise interventions have been shown to minimise decline in cardiorespiratory fitness and improve muscle strength, ${ }^{32} 3336$ physical function, ${ }^{39}$ bone mineral density, sleep ${ }^{22}$ and shoulder range of motion. ${ }^{22}{ }^{32}$ Resistance training alone in patients with breast cancer during adjuvant chemotherapy can significantly reverse sarcopaenia and dynapaenia. ${ }^{29}$

Prior exercise-based intervention studies that have included patients with breast cancer are characterised by heterogeneity, especially regarding the type, duration and intensity of exercise. ${ }^{1628} 39$ This gives rise to variations in the information on exercise interventions for this group, especially in terms of type and intensity. Therefore, the evidence-based ACSM guidelines ${ }^{20-22}$ were used for designing the intervention in this study. The intervention is based mainly on supervised PRT because of its known ability to counter the frequent side effects due to ageing, cancer and cancer treatment, especially cancerrelated cachexia. ${ }^{80}$ Cancer-related cachexia is significantly associated with impairment of ADL and poorer survival in the geriatric oncology population. ${ }^{40}$ Furthermore, breast cancer and old age are associated with an increased inflammatory profile, leading to comorbidities and decreased physical function. ${ }^{41}{ }^{42}$ Associations between inflammatory profile and exercise in older patients with breast cancer is an unexplored field ${ }^{42}$ and could lead to clinically relevant new knowledge.

Many older persons do not meet the recommended intake of $0.8 \mathrm{~g} / \mathrm{kg}$ of protein, increased to at least $1.5 \mathrm{~g} /$ $\mathrm{kg}$ in the sarcopaenic and cachectic population in their daily diet. ${ }^{9}$ Therefore, a protein supplement was included in the intervention to prevent further deficiencies in protein intake. Aerobic exercise at a light-tomoderate intensity is included in this study and measured using a pedometer which counts daily steps. To prevent increased energy demands, possibly causing weight loss, vigorous aerobic exercise is not included in this study. The absence of vigorous intensity cardiorespiratory training is in accordance with the ACSM guidelines, which state that adults should perform either moderate( $\geq 5$ days/week), vigorous ( $\geq 3$ days/week) intensity cardiorespiratory training or a combination. ${ }^{21} 22$

A possible limitation of this study could be the potential heterogenous sample. The study was primarily designed for patients in adjuvant treatment. However, due to poor recruitment, inclusion criteria were broadened to also include patients receiving neoadjuvant and metastatic treatment. It may not be possible to perform subgroup analysis on different treatments, due to lack of power.

To minimise the burden of completing multiple questionnaires, a specific questionnaire regarding fatigue was not included in this study. However, MDASI and EORTC QLQ-C30 include questions regarding fatigue

Exercise can influence a variety of recommended outcome measures of relevance to older women with breast cancer, including physical function, physical wellbeing and QoL; although few studies have included assessments of the latter two. ${ }^{16}$ Other outcome measures of importance to patients, healthcare stakeholders and practice are incidence of hospitalisation, reduction in cancer treatment toxicity and survival. ${ }^{16}$ The evidencebased intervention and a broad array of objective and patient-reported outcome measures recommended for this patient group are considered to be a strength in this study. ${ }^{16} 2122$

Exercise interventions that include older women with breast cancer receiving medical treatment is an unexplored field. The BREACE study could provide new valuable knowledge on early rehabilitation for this patient group, and the study's intervention could possibly hinder or minimise side effects, decline in physical function and improve QoL in both the short and long term in this group of patients. Should the study prove effective, the pragmatic approach to the intervention and integration in the hospital and home setting is believed to be implementable in many older patients with breast cancer.

\section{Author affiliations}

${ }^{1}$ Department of Physiotherapy and Occupational Therapy, Herlev and Gentofte University Hospital, Herlev, Denmark

${ }^{2}$ Department of Oncology, Herlev and Gentofte University Hospital, Herlev, Denmark ${ }^{3}$ Department of Oncology and Hematology, Rigshospitalet, Copenhagen, Denmark ${ }^{4}$ Department of Medicine, Herlev and Gentofte University Hospital, Herlev, Denmark ${ }^{5}$ Department of Clinical Medicine, Faculty of Health and Medical Sciences, University of Copenhagen, Kobenhavn, Denmark

${ }^{6}$ QD-Research Unit, Herlev and Gentofte Hospital, Copenhagen University Hospital, Herlev, Denmark

${ }^{7}$ Department of Physiotherapy and Occupational Therapy, Herlev and Gentofte University Hospital, Hellerup, Denmark

${ }^{8}$ Institute of Sports Science and Clinical Biomechanics, University of Southern Denmark, Odense, Denmark

${ }^{9}$ Department of Clinical Physiology and Nuclear Medicine, Herlev and Gentofte Hospital, University of Copenhagen, Herlev, Denmark

Contributors Authorship follows the Vancouver guidelines. HHA, DLN, MKM, JSJ, $\mathrm{CML}, \mathrm{AV}, \mathrm{CJ}$ and IL designed the study. AV, A-MR and IL designed the intervention. HHA and IL wrote the manuscript, with support from DLN, MKM, JSJ, AV, CJ, BZ and A-MR. All authors read and approved the final manuscript.

Funding This work was supported by the VELUX Foundation (ID number 00018310). The VELUX Foundation (or other future funding organisations) will not have any role in defining the research question, study design or protocol, data collection, interpretation of data, or in drafting or approval of coming publications. There is no commercial or politically binding relationship between any members of the TOLCA (Translational and clinical research focused on older patients with cancer) project group and the VELUX Foundation.

Competing interests None declared.

Patient consent for publication Not required.

Provenance and peer review Not commissioned; externally peer reviewed.

Open access This is an open access article distributed in accordance with the Creative Commons Attribution Non Commercial (CC BY-NC 4.0) license, which permits others to distribute, remix, adapt, build upon this work non-commercially, and license their derivative works on different terms, provided the original work is properly cited, appropriate credit is given, any changes made indicated, and the use is non-commercial. See: http://creativecommons.org/licenses/by-nc/4.0/. 
ORCID iD

Høgni Hammershaimb Andersen http://orcid.org/0000-0002-4558-8181

\section{REFERENCES}

1 Bray F, Ferlay J, Soerjomataram I, et al. Global cancer statistics 2018: GLOBOCAN estimates of incidence and mortality worldwide for 36 cancers in 185 countries. CA Cancer J Clin 2018;68:394-424.

2 Schuil H, Derks M, Liefers G-J, et al. Treatment strategies and survival outcomes in older women with breast cancer: a comparative study between the focus cohort and Nottingham cohort. $J$ Geriatr Oncol 2018;9:635-41.

3 Kenzik KM, Kent EE, Martin MY, et al. Chronic condition clusters and functional impairment in older cancer survivors: a population-based study. J Cancer Surviv 2016;10:1096-103.

4 Bellizzi KM, Mustian KM, Palesh OG, et al. Cancer survivorship and aging : moving the science forward. Cancer 2008;113:3530-9.

5 Baker F, Haffer SC, Denniston M. Health-related quality of life of cancer and noncancer patients in medicare managed care. Cancer 2003:97:674-81.

6 Mohile SG, Xian Y, Dale W, et al. Association of a cancer diagnosis with vulnerability and frailty in older medicare beneficiaries. J Natl Cancer Inst 2009;101:1206-15.

7 Cruz-Jentoft AJ, Baeyens JP, Bauer JM, et al. Sarcopenia: European consensus on definition and diagnosis: report of the European Working group on sarcopenia in older people. Age Ageing 2010;39:412-23.

8 Williams GR, Rier HN, McDonald A, et al. Sarcopenia \& aging in cancer. J Geriatr Oncol 2018.

9 Ali S, Garcia JM, Sarcopenia GJM. Sarcopenia, cachexia and aging: diagnosis, mechanisms and therapeutic options - a mini-review. Gerontology 2014;60:294-305.

10 Guigni BA, Callahan DM, Tourville TW, et al. Skeletal muscle atrophy and dysfunction in breast cancer patients: role for chemotherapyderived oxidant stress. Am J Physiol Cell Physiol 2018;315:C744-56.

11 Jensen JD, Cold S, Nielsen MH, et al. Trends in breast cancer in the elderly in Denmark, 1980-2012. Acta Oncol 2016;55:59-64.

12 Jones LW, Eves ND, Haykowsky M, et al. Exercise intolerance in cancer and the role of exercise therapy to reverse dysfunction. Lancet Oncol 2009;10:598-605.

13 Buffart LM, Kalter J, Sweegers MG, et al. Effects and moderators of exercise on quality of life and physical function in patients with cancer: an individual patient data meta-analysis of 34 RCTs. Cancer Treat Rev 2017:52:91-104.

14 Gerritsen JKW, Vincent AJPE. Exercise improves quality of life in patients with cancer: a systematic review and meta-analysis of randomised controlled trials. Br J Sports Med 2016;50:796-803.

15 Adamsen L, Quist M, Andersen C, et al. Effect of a multimodal high intensity exercise intervention in cancer patients undergoing chemotherapy: randomised controlled trial. BMJ 2009;339:b3410.

16 Kilari D, Soto-Perez-de-Celis E, Mohile SG, et al. Designing exercise clinical trials for older adults with cancer: recommendations from 2015 cancer and aging Research Group NCI U13 meeting. J Geriatr Oncol 2016;7:293-304.

17 Scher KS, Hurria A. Under-Representation of older adults in cancer registration trials: known problem, little progress. J Clin Oncol 2012;30:2036-8.

18 Slade SC, Dionne CE, Underwood M, et al. Consensus on exercise reporting template (CERT): modified Delphi study. Phys Ther 2016;96:1514-24.

19 Chan A-W, Tetzlaff JM, Gøtzsche PC, et al. SPIRIT 2013 explanation and elaboration: guidance for protocols of clinical trials. BMJ 2013;346:e7586.

20 American College of Sports Medicine, Chodzko-Zajko WJ, Proctor DN, et al. American College of sports medicine position stand. exercise and physical activity for older adults. Med Sci Sports Exerc 2009;41:1510-30.

21 American College of Sports Medicine. American College of sports medicine position stand. progression models in resistance training for healthy adults. Med Sci Sports Exerc 2009;41:687-708.

22 Schmitz KH, Courneya KS, Matthews C, et al. American College of sports medicine roundtable on exercise guidelines for cancer survivors. Med Sci Sports Exerc 2010;42:1409-26.
23 Jones CJ, Rikli RE, Beam WC. A 30-S chair-stand test as a measure of lower body strength in community-residing older adults. Res $Q$ Exerc Sport 1999;70:113-9.

24 Lønbro S, Dalgas U, Primdahl H, et al. Feasibility and efficacy of progressive resistance training and dietary supplements in radiotherapy treated head and neck cancer patients--the DAHANCA 25A study. Acta Oncol 2013;52:310-8.

25 Litterini AJ, Fieler VK, Cavanaugh JT, et al. Differential effects of cardiovascular and resistance exercise on functional mobility in individuals with advanced cancer: a randomized trial. Arch Phys Med Rehabil 2013;94:2329-35.

26 Oldervoll LM, Loge JH, Lydersen S, et al. Physical exercise for cancer patients with advanced disease: a randomized controlled trial. Oncologist 2011;16:1649-57.

27 Wright AA, Cook CE, Baxter GD, et al. A comparison of 3 methodological approaches to defining major clinically important improvement of 4 performance measures in patients with hip osteoarthritis. J Orthop Sports Phys Ther 2011;41:319-27.

28 Lahart IM, Metsios GS, Nevill AM, et al. Physical activity for women with breast cancer after adjuvant therapy. Cochrane Database Syst Rev 2018;1:Cd011292.

29 Adams SC, Segal RJ, McKenzie DC, et al. Impact of resistance and aerobic exercise on sarcopenia and dynapenia in breast cancer patients receiving adjuvant chemotherapy: a multicenter randomized controlled trial. Breast Cancer Res Treat 2016;158:497-507.

30 Cadmus LA, Salovey P, Yu H, et al. Exercise and quality of life during and after treatment for breast cancer: results of two randomized controlled trials. Psychooncology 2009;18:343-52.

31 Carayol M, Bernard P, Boiché J, et al. Psychological effect of exercise in women with breast cancer receiving adjuvant therapy: what is the optimal dose needed? Ann Oncol 2013;24:291-300.

32 Casla S, López-Tarruella S, Jerez Y, et al. Supervised physical exercise improves VO2max, quality of life, and health in early stage breast cancer patients: a randomized controlled trial. Breast Cancer Res Treat 2015;153:371-82.

33 Courneya KS, Segal RJ, Mackey JR, et al. Effects of aerobic and resistance exercise in breast cancer patients receiving adjuvant chemotherapy: a multicenter randomized controlled trial. J Clin Oncol 2007;25:4396-404.

34 Schmidt ME, Wiskemann J, Armbrust P, et al. Effects of resistance exercise on fatigue and quality of life in breast cancer patients undergoing adjuvant chemotherapy: a randomized controlled trial. Int J Cancer 2015;137:471-80.

35 Schmidt T, Weisser B, Dürkop J, et al. Comparing endurance and resistance training with standard care during chemotherapy for patients with primary breast cancer. Anticancer Res 2015;35:5623-9.

36 Travier N, Velthuis MJ, Steins Bisschop CN, et al. Effects of an 18-week exercise programme started early during breast cancer treatment: a randomised controlled trial. BMC Med 2015;13:121.

37 van Vulpen JK, Schmidt ME, Velthuis MJ, et al. Effects of physical exercise on markers of inflammation in breast cancer patients during adjuvant chemotherapy. Breast Cancer Res Treat 2018;168:421-31.

38 van Waart $\mathrm{H}$, Stuiver MM, van Harten WH, et al. Effect of lowintensity physical activity and moderate- to high-intensity physical exercise during adjuvant chemotherapy on physical fitness, fatigue, and chemotherapy completion rates: results of the PACES randomized clinical trial. J Clin Oncol 2015;33:1918-27.

39 Gebruers N, Camberlin M, Theunissen F, et al. The effect of training interventions on physical performance, quality of life, and fatigue in patients receiving breast cancer treatment: a systematic review. Support Care Cancer 2019;27:109-22.

40 Dunne RF, Roussel B, Culakova E, et al. Characterizing cancer cachexia in the geriatric oncology population. J Geriatr Oncol 2019;10:415-9.

41 Monteiro-Junior RS, de Tarso Maciel-Pinheiro P, da Matta Mello Portugal E, et al. Effect of exercise on inflammatory profile of older persons: systematic review and meta-analyses. J Phys Act Health 2018;15:64-71.

42 de Jesus Leite MAF, Puga GM, Arantes FJ, et al. Effects of combined and resistance training on the inflammatory profile in breast cancer survivors: a systematic review. Complement Ther Med 2018;36:73-81. 